\title{
NEEDLESCOPIC SURGERY FOR PALMAR HYPERHIDROSIS
}

Hidehiro Yamamoto, $\mathrm{MD}^{\mathrm{a}}$ Akio Kanehira, MD $^{\mathrm{b}}$

Munenori Kawamura, $\mathrm{MD}^{\mathrm{c}}$

Masayoshi Okada, MD

Yutaka Ohkita, MD
Objective: The aim of this study was to develop a less invasive technique for video-assisted thoracic sympathectomy.

Methods: A newly designed trocar was used. A skin incision of 2.0 to $2.5 \mathrm{~mm}$ in length was made in the third or fourth intercostal space at the midaxillary line. After insertion of the trocar, thoracic sympathectomy with electrocautery was carried out at the level of the second and third ribs.

Results: From October 1998 to March 1999, 180 patients with palmar hyperhidrosis underwent this technique. No complications related to the technique occurred, and within 1 week the operative wound had almost completely disappeared. There were recurrences necessitating reapplication of the technique.

Conclusion: The technique allowed healing without a scar and improved the patient's cosmesis. (J Thorac Cardiovasc Surg 2000;120:276-9)
V deo-assisted thoracic sympathectomy (VATS) is the most effective treatment for palmar hyperhidrosis, and results are less invasive and offer good cosmesis. ${ }^{1-4}$ Patients with palmar hyperhidrosis are estimated to represent $1 \%$ of the Asian population. In patients with palmar hyperhidrosis, VATS has usually been carried out with more than two skin incisions or with a large thoracoscope of more than $10 \mathrm{~mm}$ in diameter through a single surgical port. Until now, patients have had to be content with operative scars. ${ }^{5}$ This study describes a new VATS technique, termed needlescopic surgery, that allows healing without a scar.

\section{Methods}

Between October 1998 and March 1999, we treated 180 patients with palmar hyperhidrosis by using this new technique. The mean age of patients was 28.2 years (range, 8-56 years). Eighty-four patients were male, and 96 were female. Each patient's chest roentgenogram was examined. Any

From the Department of Surgery, Division II, Kobe University School of Medicine, ${ }^{a}$ the Department of Surgery, Hyogo Hospital, ${ }^{\text {b }}$ and the Department of Surgery, Kyowa Hospital, ${ }^{c}$ Kobe, Japan

Received for publication Oct 27, 1999; revisions requested Feb 2, 2000; revisions received March 20, 2000; accepted for publication April 12, 2000.

Address for reprints: Hidehiro Yamamoto, MD, Department of Surgery, Division II, Kobe University School of Medicine, 7-5-2 Kusunoki-chou, Chuoku, Kobe, Japan 650 (E-mail: hideyama@mua.biglobe.ne.jp).

Copyright (c) 2000 by The American Association for Thoracic Surgery

$0022-5223 / 2000 \$ 12.00+0 \quad \mathbf{1 2 / 1 / 1 0 7 8 3 0}$

doi: $10.1067 / \mathrm{mtc} .2000 .107830$ abnormal shadow, including pleural thickening, was considered a contraindication to this new VATS technique.

Equipment used consisted of a thoracoscope $2 \mathrm{~mm}$ in diameter (2MM MiniSite Gold Laparascope, United States Surgical Corporation, Norwalk, Conn), a newly designed trocar (scope sheath) that we produced (Fig 1), a digital monitor system, and standard endoscopic instruments. A high-resolution video monitor and the remaining operative setup was similar to that used in standard video-assisted thoracic operations.

Patients were placed in a sitting position and given a general anesthetic through a double-lumen endotracheal tube. Onelung ventilation was used while the procedure was performed. A skin incision of 2.0 to $2.5 \mathrm{~mm}$ was made on the third or fourth intercostal space on the midaxillary line (Fig 2). The best location for cosmesis of the skin incision was determined preoperatively. While the trocar was inserted, saline solution or air was passed through the internal tube by means of a 10 $\mathrm{mL}$ syringe. When the tip of the trocar reached the pleural cavity, loss of resistance to the syringe was felt. Then the internal tube was removed, and the thoracoscope was inserted. When observation was needed, the tip of the scope was positioned slightly ahead of the external sheath. After the absence of adhesions was verified, the inner surface of the chest wall was reached by advancing the scope sheath. We identified the sympathetic trunk by using anatomic landmarks, including the azygos vein and the first and second ribs with their costovertebral junctions. A fluoroscope was used to determine the sympathetic level.

When application of treatment was intended, the tip of the scope was positioned slightly behind the sheath tip. Then the tip of the scope sheath became visible on the monitor. Visualization was maintained through the internal lumen of the scope and the two windows of the scope sheath. Even when the trunk was not clearly visible, the tip of the scope sheath could be applied to the presumed location of the trunk at the region of the rib head of the second, third, and fourth ribs. The trunk 


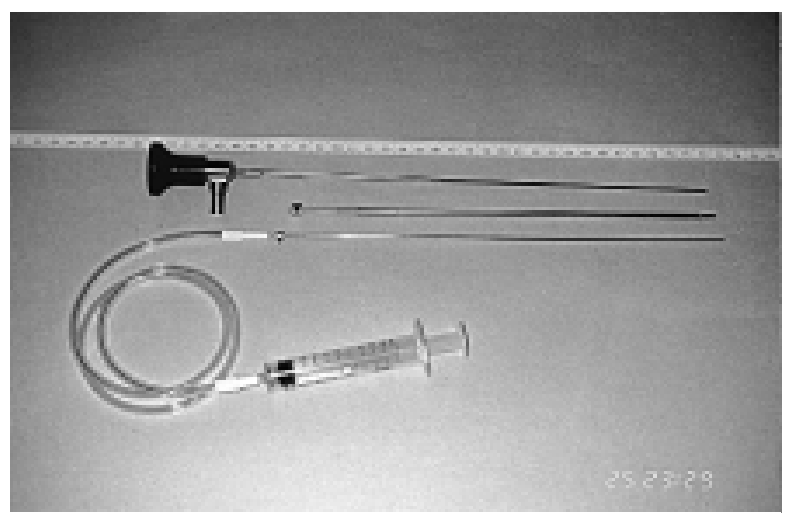

Fig 1. The new trocar (N5C scope guide) consists of an internal tube and an external tube or sheath. The internal tube has an internal diameter of $1 \mathrm{~mm}$ and is used with a $10-\mathrm{mL}$ syringe. The tip of the internal tube is polished round to prevent injury to the lung on puncture of the chest wall. The external diameter of the external sheath is $2.5 \mathrm{~mm}$. The external surface is coated with an insulating membrane, except for the tip and the end. Since this device is made from stainless steel, it can be used as an electro-probe to search for the sympathetic trunk when the trunk is invisible. Sympathectomy with electrocautery can also be performed through the external pipe without a short circuit. Since there are two windows in the tip, smoke can be expelled, making visualization possible during electrocautery.

can also be located by means of palpation because it is a distinct vertical cordlike structure overlying the rib head region.

Identification of the sympathetic segment was confirmed with the aid of its vasomotor response to electrical stimulation and by evaluating the change in microcirculation on the skin of the patient's finger with a laser Doppler flowmeter (PeriFlux PF 4001 flowmeter; Perimed AB, Stockholm, Sweden). A transient decrease of the microcirculation without a change in the temperature of the finger in response to test electrocoagulation indicated that the proper sympathetic segment was being stimulated, including the occult nerve of the Kuntz branch. ${ }^{6,7}$ The coagulation power at a low control setting was equivalent to 0.05 to $0.08 \mathrm{~A}$ from a Mera electrosurgical unit, (MS7000SAS, Senko Medical Manufacturing Co, Ltd, Tokyo, Japan). Subsequently, a stronger electric current of 0.08 to $0.2 \mathrm{~A}$ was applied to help dissect the sympathetic trunk on the second and third ribs.

A continual increase in microcirculation, along with a gradual elevation in finger temperature, indicated that the lesion had been made in the correct place. Usually an increase of microvascular perfusion and an elevation of temperature of more than $50 \%$ above the original level was noted, as measured by means of the laser Doppler perfusion unit. This change was interpreted as a consequence of vasodilation resulting from sympathetic denervation. ${ }^{7}$ At this point, the sympathectomy was considered complete. The absence of air leaks and bleeding was confirmed. The tip of the scope sheath

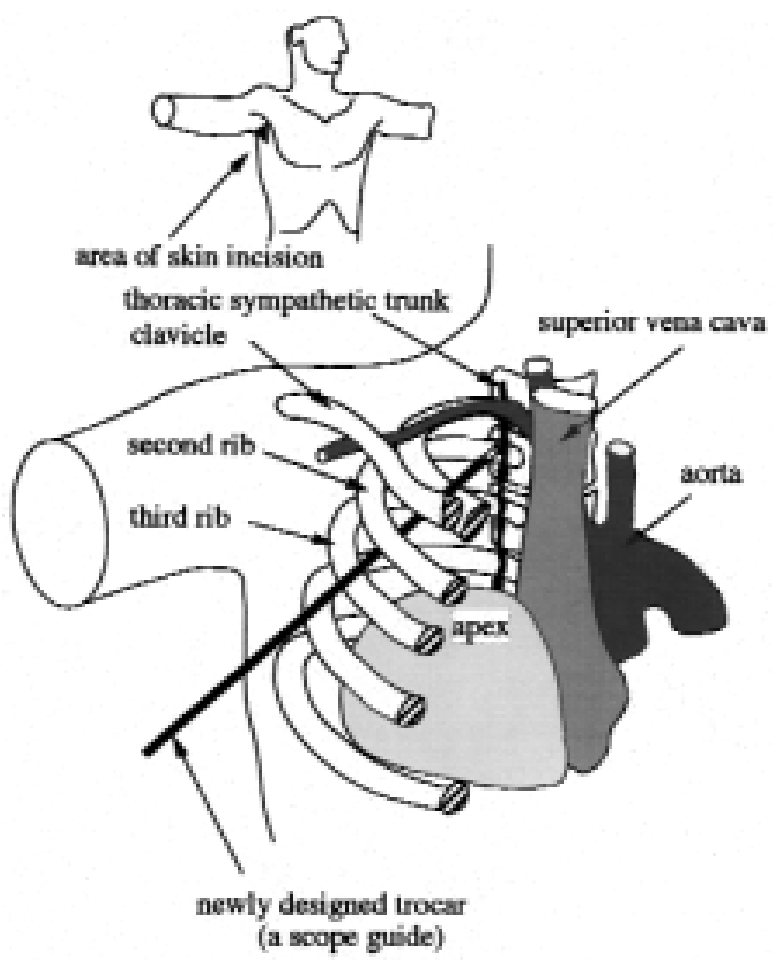

Fig 2. A schematic operative illustration providing an intraoperative view of this technique. When the scope was withdrawn slightly from the external tube or sheath, the tip of the latter came into view. The visual field of the scope is not disturbed by the sheath, even when the tip of the sheath is positioned behind the tip of the scope. The view is aided in this position by the presence of the two windows of the scope sheath. The tip comes into contact with the thoracic sympathetic trunk, and sympathectomy is carried out by electrocautery.

was positioned in the apex of the pleural cavity, and the lung was inflated by the anesthetist. Air in the pleural space was expelled through the lumen of the scope sheath, and the scope itself was then removed from the scope sheath. Sufficient expansion of the lung was confirmed with a second placement of the scope, and then the scope and scope sheath were fully removed. Thoracotomy tubes were not used, and the wound was closed with sterile surgical tape.

Follow-up was recorded on return visits or by telephone, fax, or E-mail at 3 months, 6 months, and 1 year.

\section{Results}

Mean operative time was $15.0 \pm 3.5$ minutes for one side and $27 \pm 3.1$ minutes for both sides. Mean anesthesia time was $37 \pm 5.6$ minutes. Pneumopleural adhesions were observed in one patient, but these adhesions were dissected by use of a trocar and electrocautery. None of the patients needed to undergo a different type 
of surgical technique. In all patients the wound usually closed by itself. Operative bleeding was minimal $(<1$ $\mathrm{mL}$ ). None of the patients had a postoperative pneumothorax. One hundred forty-five patients were discharged on the day of the operation, and the remaining 35 returned home the day after their operation. The excess sweating of the hands stopped as intended, and Horner syndrome did not occur. The postoperative follow-up period ranged from 6 to 12 months, and the efficacy of this technique was $100 \%$ at 6 months and 98.3\% at 10 months. Three patients had a relapse of $50 \%$ to $70 \%$ compared with their preoperative condition. These patients underwent a second operation 14 months after the first operation, and the intraoperative findings showed that the sympathetic trunk was scarred. Electrocautery to the trunk was again performed on the second and third ribs. After this second procedure, palmar hydrosis was completely cured in all 3 patients.

One hundred fifteen $(63.9 \%)$ patients reported mild or moderate compensatory sweating of the back, abdomen, thigh, breast, face, and axillae. For all of these patients, this was tolerable. One patient complained of severe compensatory sweating of the back and thighs. The other 64 patients reported minimal or no compensatory sweating. Gustatory sweating was observed in only 7 (3.9\%) patients. There was no incidence of wound pain, numbness, or cosmesis. The wounds almost completely disappeared within 1 week of the procedure. There were no scars, except in 7 cases with linear pigmentation of $0.6 \times 2.0 \mathrm{~mm} 2$ months after the operation.

\section{Discussion}

The merit of this technique is that VATS can be performed easily with a clear and magnified visual field and a short operative time. Because white smoke, which is created by the electrocautery process, can be expelled through the two windows of the external tube, a clear view on the monitor can be maintained during the entire procedure. Meticulous observation and fine dissection of the target can be done because of the magnified view through the thoracoscope. This technique allows the surgeon to divide the sympathetic chain into halves. Furthermore, dissection can always be done at the center of the monitor because the scope is always firmly held in the center of the scope sheath.

VATS is in widespread use as a less-invasive technique than conventional thoracotomy. The final evolution of a less invasive technique is the so-called needlescopic surgery, as reported here.
Needlescopic surgery has several implications. One is that the number of skin incisions is reduced to one, and the incision itself is as small as a needle point. VATS has usually been done with more than two surgical ports, except for a technique that we previously reported using a flexible scope. ${ }^{8,9}$ A greater number and length of skin incisions adds to the surgical trauma and devalues the operation. The length of the wound is also a matter of the greatest importance for cosmesis. The 3.4-mm trocar (Mini-site Inducer, United States Surgical Corporation) is popular as the smallest trocar available. With the 3.4-mm trocar, 4 skin incisions must be made bilaterally. These incisions remain as scars, whereas the new technique allows for a thoracic sympathectomy through a skin incision of 2.0 to 2.5 $\mathrm{mm}$. A further benefit of this technique is that the skin incision can be made in an inconspicuous location, such as the armpit.

The reason for the patient to be in a sitting position with one-lung ventilation is that no postural change is required to perform this technique. Some surgeons perform a thoracic sympathectomy with the patient in a semirecumbent position because the apex of the lung falls away from the chest wall as soon as the single lumen tube is disconnected, and this provides visualization of the dorsal sympathetic chain. We judged that the total anesthesia time when 1-lung ventilation and a sitting position were used was shorter overall.

There is a potential risk when a thoracotomy is performed through a small skin incision. The presence of severe pneumopleural adhesions increases the possibility of lung injury. However, the risk of this technique is thought to be the same as that accompanying the use of the 10-mm trocar, and our results showed that such a risk was not high. As for side effects, compensatory sweating was moderate, and the frequency did not differ from the results of our previous technique. ${ }^{8,9}$ The reported incidence of compensatory hyperhidrosis is $63 \%$ to $81 \%$ by the supraclavicular approach ${ }^{10,11}$ and $82 \%$ by thoracic endoscopic approach. ${ }^{12,13}$ The reported incidence of recurrence was $24(2.1 \%)$ in 1163 patients after a simple endoscopic technique with standard urologic equipment. ${ }^{14}$ Another report showed that the operative failure was 4 (1.9\%) in 270 patients with palmar hyperhidrosis, and recurrence was $4 \%$ after more than 9 months postoperatively. ${ }^{15}$ The results of the new technique were thought to compare favorably with those reported in previous studies.

In conclusion, it is obviously important to the patients that a thoracic sympathectomy results in only minimal scarring because palmar hyperhidrosis is treat- 
ed with the intent of improving the patient's quality of life. Poor cosmesis detracts from the value of VATS as a way of treating palmar hyperhidrosis, a benign disease. We believe that needlescopic surgery successfully addresses these issues.

We thank Yoshimasa Nishio, MD, and Hidetugu Sekino, $\mathrm{MD}$, for operative assistance.

\section{REFERENCES}

1. Shachor D, Jedeikin R, Olsfanger D, et al. Endoscopic transthoracic sympathectomy in the treatment of primary hyperhidrosis. Arch Surg 1994;129:241-4.

2. Josephs LG, Menzoiam JO. Technical considerations in endoscopic cerviothoracic sympathectomy. Arch Surg 1996;131:355-9.

3. Drott C, Claes G. Hyperhidrosis treated by thoracoscopic sympathicotomy. Cardiovasc Surg 1996;4:788-90.

4. Rex LO, Drott C, Claes G, et al. The Boras experience of endoscopic thoracic sympathicotomy for palmar, axillary, facial hyperhidrosis and facial blushing. Eur J Surg Suppl 1998;580:23-6.

5. Yuan RH. Mini-laparoscopic cholecystectomy: a cosmetically better, almost scar-less procedure. J Laparoendosc Adv Surg Tech A 1997; 7:205-11.

6. Kuntz A. Distribution of the sympathetic rami to the brachial plexus: its relation to sympathectomy affecting the upper extremity. Arch Surg 1927;15:871-7.

7. Kau M-C. Video endoscopic sympathectomy using a fiberoptic CO2 LAZER to treat palmar hyperhidrosis. Neurosurgery 1992;30:131-5.

8. Yamamoto H, Okada M, Takada M, et al. Video-assisted thoracic surgery through a single skin incision. Arch Surg 1998;133:145-7.

9. Yamamoto H, Okada M, Kanehira A, et al. Clinical experience of video-assisted thoracic sympathectomy through the retrosternal pulmonary junction. Ann Thorac Surg 1999;68:2361-3.

10. Adar R, Kurchin A, Zweig A, et al. Palmar hyperhidrosis and its surgical treatment. Ann Surg 1977;186:34-41.

11. Bass A, Inovrotzlavski S, Adar R. Upper dorsal sympathectomy for hyperhidrosis. Isr J Med Sci 1983;19:112-5.

12. Chou SH, Lee SH, Kau E-L. Thoracic endscopic T2-T3 sympathectomy in palmar hyperhidrosis: experience of 112 cases. Surg Today 1993;23:105-7.

13. Edmondson RA, Bannerjee AK, Rennie JA. Endoscopic tranthoracic sympathectomy in the treatment of hyperhidrosis. Ann Surg 1992;215:289-93.

14. Drott C, Claes G. Hyperhidrosis treated by thoracoscopic sympathectomy. Cardiovasc Surg 1996;4:788-91.

15. Herbst F, Plas EG, Függer R, et al. Endoscopic thoracic sympathectomy for palmar hyperhidrosis of the upper limbs. Ann Surg 1994;220:86-90. 